SUBPART TWELVE

Other Modules 



\title{
Evacuation Planning: An Integrated Approach
}

\author{
Gregor Lämmel, Christoph Dobler and Hubert Klüpfel
}

\subsection{Basic Information}

\section{Entry point to documentation:}

http://matsim.org/extensions $\rightarrow$ evacuation

\section{Invoking the module:}

http://matsim.org/javadoc $\rightarrow$ evacuation $\rightarrow$ RunEvacuationExample class

\section{Selected publications:}

Lämmel (2011); Lämmel et al. (2009)

This chapter presents an integrated approach for performing evacuation simulations with MATSim using the evacuation contribution. The approach comprises all workflow steps for performing an evacuation analysis: i.e., selecting the evacuation area and defining the population, specifying behavioral parameters (i.e., pre-movement time distribution and mode of evacuationcar or pedestrian) and analyzing the simulation output. These steps can all be performed within one graphical user interface. Additionally, two extensions of MATSim for simulating public transport and changing the network during simulation (i.e., network change events) are accessible from the GUI. In this chapter, the steps for performing such an integrated analysis are described and illustrated based on the Hamburg-Wilhelmsburg example. A detailed case study based on this scenario is given in Chapter 71, as well as in Durst et al. (2014); Hugenbusch (2012).

\subsection{Related Work}

Simulation of evacuation processes has attracted much attention in recent decades; reasons include increases in frequency and severity of natural hazards jeopardizing various populations

How to cite this book chapter:

Lämmel, G, Dobler, C and Klüpfel, H. 2016. Evacuation Planning: An Integrated Approach. In: Horni, A, Nagel, K and Axhausen, K W. (eds.) The Multi-Agent Transport Simulation MATSim, Pp. 271-282. London: Ubiquity Press. DOI: http://dx.doi.org/10.5334/baw.41. License: CC-BY 4.0 
and regions, as well as (social) disasters (Rodríguez et al., 2006). Another factor is the availability of large-scale, fast simulation models and tools. Lämmel (2011) discusses such a model employed as a contribution to MATSim. Basically, this model implements the same iterative learning approach as that applied to "regular" transport scenarios. In the first instance, the cost function comprises only travel times, albeit a combination of travel time and travel distance; as a cost function has been investigated as well (Lämmel et al., 2009). Artificial agents represent evacuees trying to improve their evacuation plans from iteration to iteration, by creating new evacuation plans more responsive to the evolving situation. A typical simulation run comprises 500-1 000 iterations. The model is applied to a tsunami-related evacuation of the City of Padang in Indonesia (e.g., Taubenböck et al., 2013; Goseberg et al., 2013); some scenario details are discussed in Chapter 76.

Additional evacuation simulation related work in MATSim is presented by Dobler (2013). The main difference to this chapter's approach is that agents are allowed to adapt their plans spontaneously, using MATSim's within-day replanning framework (Dobler et al., 2012) (Chapter 30). Based on a behavioral model, agents coordinate their actions on a household level. If a household is, e.g., not complete when the evacuation starts, each member estimates time needed to return home, as well as the time required to leave the actual evacuation area. Then, the household decides whether meeting at home and leaving together is preferable to each member leaving on its own. Since the behavioral model is implemented on an agent, respectively household level, individual attributes such as children present in the household, or availability of a car, can be taken into account. In contrast to regular MATSim simulations, only a single iteration is performed. Since the agents can optimize their plans continuously using real time information, no further replanning is necessary. As a result, agents do not foresee future events like traffic jams caused by people leaving the threatened area.

An independent evacuation scenario, not using the evacuation, is presented in Chapter 60.

The remainder of this chapter is organized as follows: Section 41.3 gives a brief description on how to set up and run evacuation. A short start guide for evacuation is presented in Section 41.4. Obtaining the required input data is discussed in Section 41.5. Detailed instructions on how to use evacuation's ScenarioManager, running simulations and analysis is given in Section 41.6. This chapter concludes with a brief outlook in Section 41.7.

\subsection{Download MATSim and Evacuation}

Although the MATSim version 0.6 .0 -SNAPSHOT is referred to here, the package should also work with later versions of MATSim.

1. Download the current nightly build of MATSim and evacuation from http://matsim.org/ files/builds/.

2. Unzip the Matsim_rxxxxx.zip, Matsim_libs.zip and evacuation-0.6.0-SNAPSHOT-rxxxxx.zip.

3. Move the evacuation-0.6.0-SNAPSHOT-rxxxxx.jar and libs folder from the evacuation - 0.6.0-SNAPSHOT-rxxxxx directory one level up, i.e., to the directory, where MATSim_rxxxxx .jar is located.

Test configuration by invoking

java -cp evacuation-0.6.0-SNAPSHOT.jar;MATSim_rxxxxx.jar

org.matsim. contrib. evacuation. scenariomanager. ScenarioManager

(It is advisable to copy that command to a file evacuation . bat - or evacuation. sh, if using a Unixlike operation system. One can then run that file instead of typing the command.) 


\subsection{The Fifteen-Minute Tour}

For just a quick impression, the following steps can be performed within a few minutes:

OSM Go to http: //www. openstreetmap.org, search for the desired place and download a (small) OSM file. Please choose a small area, e.g., 500 meters by 500 meters; this is sufficient to begin and size of the exported area is limited. For larger areas, a direct download from sites like http://www. geofabrik. de is preferable (see next section).

Run the ScenarioManager as described in the previous section.

Create a scenario by clicking the leftmost button first and then New. Go to the directory where the designated project should be saved and name the project file (e.g., london.xml or scenario. $\mathrm{xml}$ ).

Specify the path of the OSM file (by clicking Set next to network) and the output directory. Leave area and population file as it is, evacuation will handle this. This step must be performed only once. After the scenario-file has been saved, one can open it in the ScenarioManager.

Sample size Set the sample size to 0.1, using the mouse or the cursor buttons on your keyboard.

Departure Specify the departure time distribution. Plausible values are: normal distribution, $\mu$ and $\sigma 600$ seconds ( 10 minutes), earliest 300 seconds, latest 1200 seconds (20 minutes).

Save the scenario file.

Area Switch to the area tab. One can define the circular evacuation area by keeping the left mouse button pressed and defining the center and radius. Do not forget to save changes.

Population Switch to the population tab and define the population (handling is similar to area). Do not forget to save changes.

Convert Switch to the next tab and convert the scenario to MATSim input files by clicking the run button. The MATSim files will be stored in the output directory specified in the beginning.

Run the MATSim simulation by skipping the next two tabs/buttons (road closures and buses) and switching to the simulation tab (with the "M" for MATSim on the computer screen). Click run. This will take a while. If an output directory (e.g., from a previous run) already exists, it will be renamed.

Analyze your simulation results by switching to the final tab after the simulation is finished.

\subsection{Input Data (any Place and any Size)}

The only external input necessary for performing an evacuation analysis with org.matsim. contrib.evacuation is an OSM file. In this tutorial, we will use the file for Hamburg, Germany. Please go to http://download.geofabrik.de/europe/germany/hamburg.html and download the hamburg-latest.osm.bz2 file. This is the only initial preparation needed. Everything else can be done with the Scenariomanager of the GUI.

\subsection{Scenario Manager}

The scenario setup, evacuation simulation, and analysis are handled by the ScenarioManager from the MATSim contribution package org.matsim. contrib. evacuation.

\subsubsection{Scenario Configuration}

At startup, the ScenarioManager offers the option to either: define a new scenario configuration or open an existing one from a XML file, which then can be modified. Figure 41.1 shows a screenshot of a scenario configuration in the ScenarioManager and the corresponding XML file, respectively. 


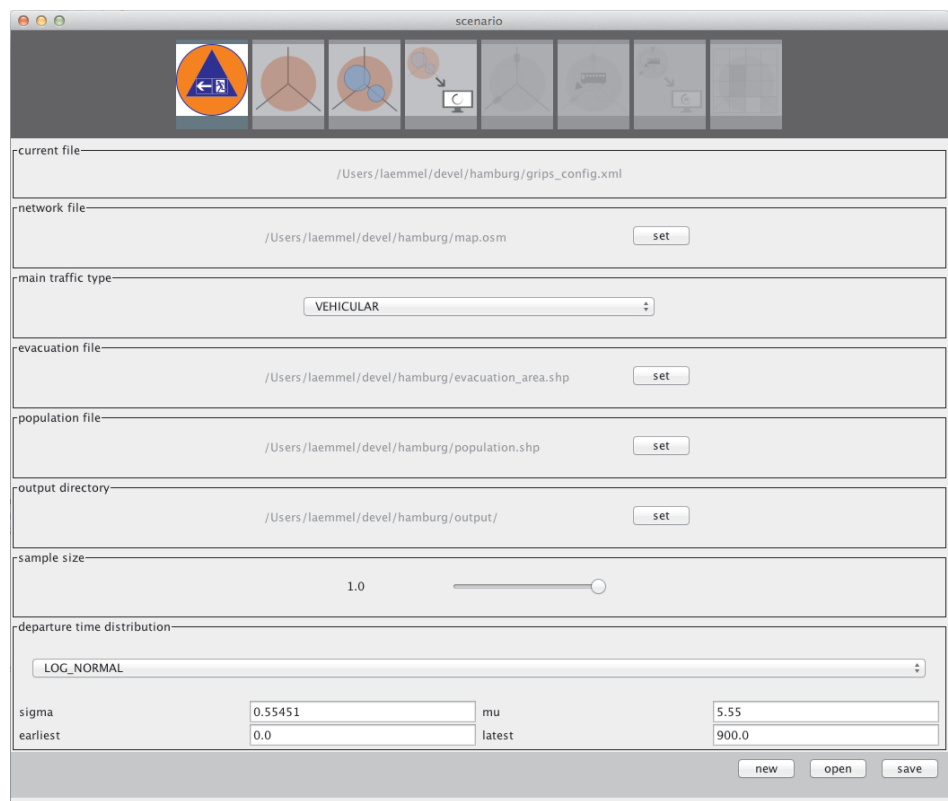

(a) ScenarioManager.

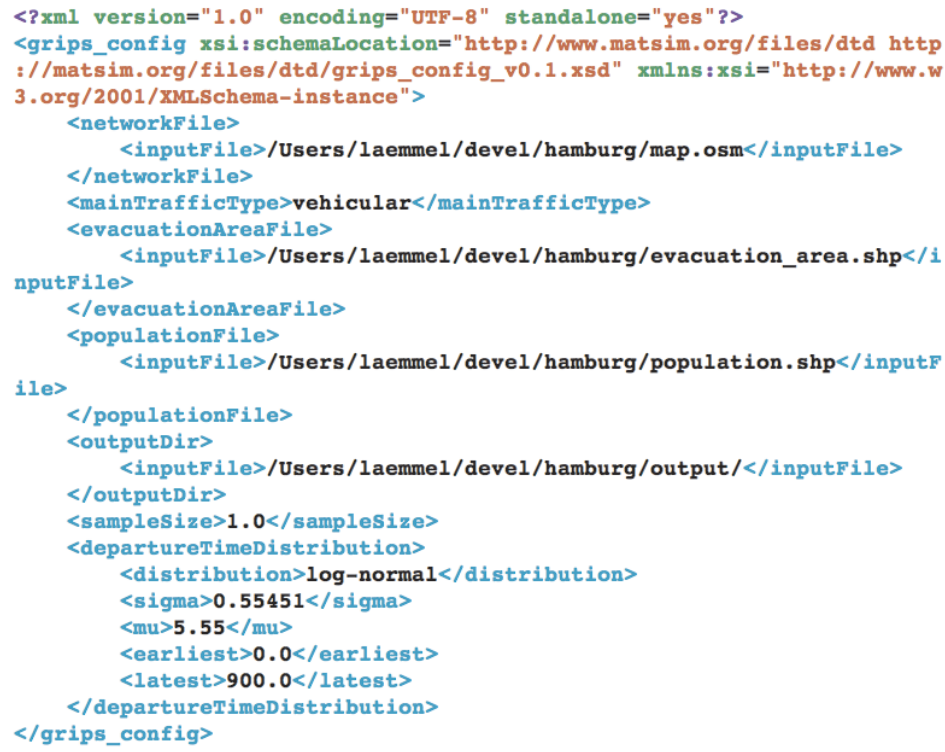

(b) XML file.

Figure 41.1: Illustration of a configuration opened in the ScenarioManager and as XML file.

The evacuation scenario is specified by the following parameters:

- The path to the network file covering the evacuation area: Currently, OSM XML files are supported (*.osm).

- The main traffic type for the simulation: This can either be: VEHICULAR or PEDESTRIAN. Depending on the choice, a vehicular specific (the MATSim default) or a pedestrian-specific 
(as discussed in Lämmel et al. (2009); Lämmel (2011)) simulation network will be generated by setting free speed, number of lanes and flow capacity for all links in the network.

- The path to a ESRI shape file describing the extent of the evacuation area, depicted by a simple polygon. This file does not have to be in place right from the beginning; it can be produced manually by the ScenarioManager itself, as discussed later.

- The path to an ESRI shape file detailing the size and distribution of the affected population. This file comprises a set of simple polygons; each polygon has an additional attribute for the number of persons residing at a location inside that polygon. The evacuation area file can be produced with help of the ScenarioManager.

- The path to the output directory where the simulation output and MATSim scenario files will be stored.

- The sample size for the MATSim simulation. A smaller sample size increases the simulation performance, while a larger size might increase accuracy of the results. Typical values are 1.0, 0.1 , or 0.01 , depending on the scenario and available computing resources.

- Departure time distribution defines the distribution from which departure times for the simulation will be drawn, based on the premise that, in real evacuation situations, all participants probably do not start evacuation simultaneously. People tend to perform pre-evacuation activities before they start, including: picking up relatives, packing food, clothes, valuable belongings, etc. Since number and duration of these activities differs by individual, population departure times are unknown quantities. The ScenarioManager supports three different distributions: (Dirac-delta, normal, and log-normal). If the user chooses the Dirac-delta distribution, then all evacuees will start simultaneously, which might be the worst case (Lämmel and Klüpfel, 2012). By choosing the normal distribution, departure times for individuals are drawn from a normal distribution with mean $\mu$ and standard deviation $\sigma$, where the parameters $\mu$ and $\sigma$ are given in seconds. As an example, setting $\mu=1800$ and $\sigma=900$ will result in a departure time distribution where, on average, after 30 minutes $50 \%$ of the population has departed and $68.3 \%$ of the population departs in time intervals of 30 minutes \pm 15 minutes. If the user chooses log-normal as the distribution, departure times are drawn from a log-normal distribution, where $\mu$ and $\sigma$ are the parameters of the associated normal distribution (a discussion on this matter is given below). The parameters earliest and latest determine the earliest and latest possible departure time. The normal and log-normal departure time distribution are truncated accordingly.

The departure time distribution is perhaps the most tenuous parameter to set; the authors found no holistic research into this matter. In general, it seems reasonable to assume that many people start evacuating at the same time, or soon after the evacuation order has been issued and as time proceeds, fewer and fewer people are left to depart. This requires a departure time distribution that has a probability density function beginning with a steep positive gradient, leveling out slowly after a peak. The probability density function of a log-normal distribution produces this kind of curve; log-normal and normal distributions are closely related. If the random variable $Y$ is normal distributed, then $X=\exp (Y)$ is log-normal distributed. The expected value $E[X]$ and the variance $\operatorname{Var}[X]$ are

$$
E[X]=\exp \left(\mu+\frac{\sigma^{2}}{2}\right)
$$

and

$$
\operatorname{Var}[X]=\exp \left(2\left(\mu+\sigma^{2}\right)\right)-\exp \left(2 \mu+\sigma^{2}\right)
$$


Conversely, if the expected value and variance is given, $\mu$ and $\sigma$ of the associated normal distribution can be obtained as follows:

$$
\sigma=\sqrt{\log \left(1+\frac{\operatorname{Var}[X]}{(E[X])^{2}}\right)}
$$

and

$$
\mu=\log \left(E[X]-\frac{1}{2} \sigma^{2}\right) .
$$

If users wish to generate a population with departure times following a log-normal distribution, it is hard to see how $\sigma$ and $\mu$ will determine the outcome. It is much more convenient to consider expected value and variance. Given Equation (41.3) and Equation (41.4), a conversion from expected value and variance to $\sigma$ and $\mu$ is straightforward.

\subsubsection{Evacuation Area}

The ScenarioManager integrates modules for the evacuation area definition and distribution of the affected population. The so-called evacuation area selection module allows the user to define the evacuation area by drawing either a simple polygon or circle on a map. The application can make use of either a WMS-provider or a tile map provider (e.g., OSM) as background map renderer. Zooming and panning is restricted to the bounding box of the OSM network file provided in the scenario configuration. An illustration of the evacuation area selector is given in Figure 41.2. In addition to defining a new evacuation area, a pre-existing one can also be loaded into the ScenarioManager. The requirements for a pre-existing evacuation area file are:

- It has to be provided as a ESRI shape file.

- The evacuation area must be defined as a simple polygon or a multi-polygon containing one, and only one, simple polygon.

- The coordinate reference system for polygon in the ESRI shape file must be set correctly.

Due to the high likelihood of error, this approach is recommended for experienced users only.

Later in the process, the ScenarioManager takes the evacuation area to cut out an evacuation network. However, after cutting out the evacuation net, there is no particular node as a target for the route calculation, as evacuees have more than one safe place as a destination. Instead, in the underlying domain, every node outside the evacuation area is a possible destination for an evacuee seeking an escape route. Thus, the evacuation problem is, in general, a multi-destination problem. To resolve this, the standard approach (e.g., Ford and Fulkerson (1962); Lu et al. (2005)) is to extend the network in the following way: All exit links (i.e., links that originate inside the evacuation area and terminate outside the evacuation area) are connected, using virtual links with very high (essentially infinite) flow capacity and equal length, to a super-node; all evacuation routes are routed to the super-node. This way, the problem is reduced to a multi-source single-destination problem. And thus, finding the shortest path from any node inside the evacuation area to this super-node and, in consequence, to safety, can efficiently be solved. For technical reasons, a superlink is added to the super-node and the evacuees are routed to that link (see the image at the beginning of this chapter).

\subsubsection{Evacuation Demand}

The process of defining the population distribution is similar to that of the evacuation area, differing because population is distributed over circles drawn on the map. The user can draw 


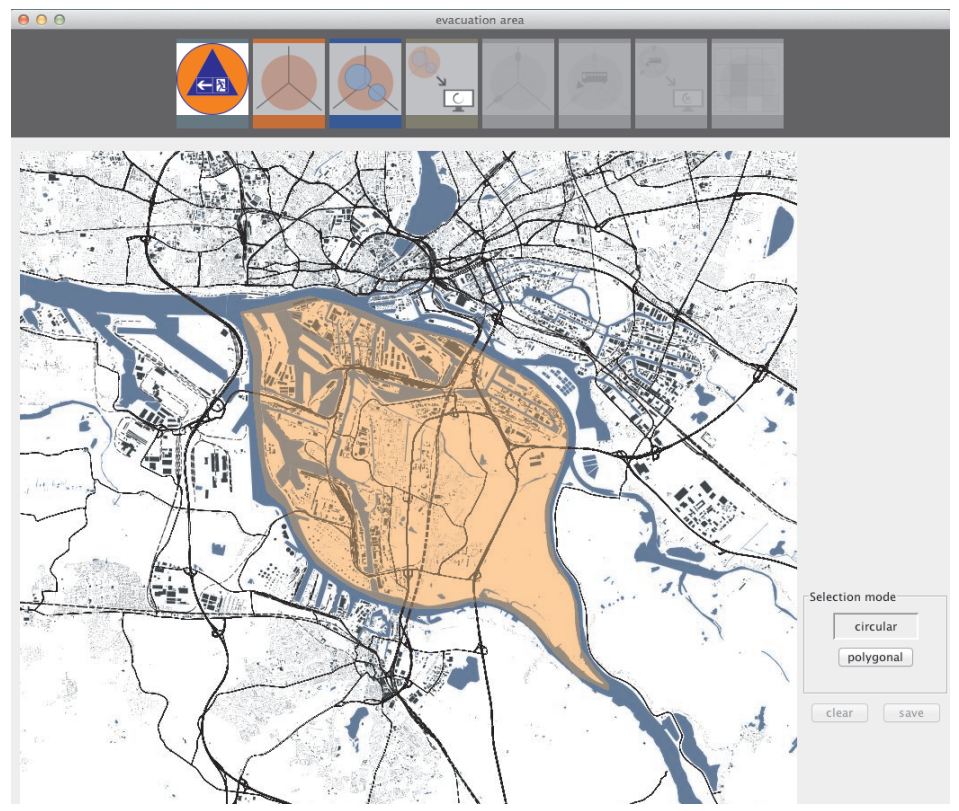

(a) Evacuation area.
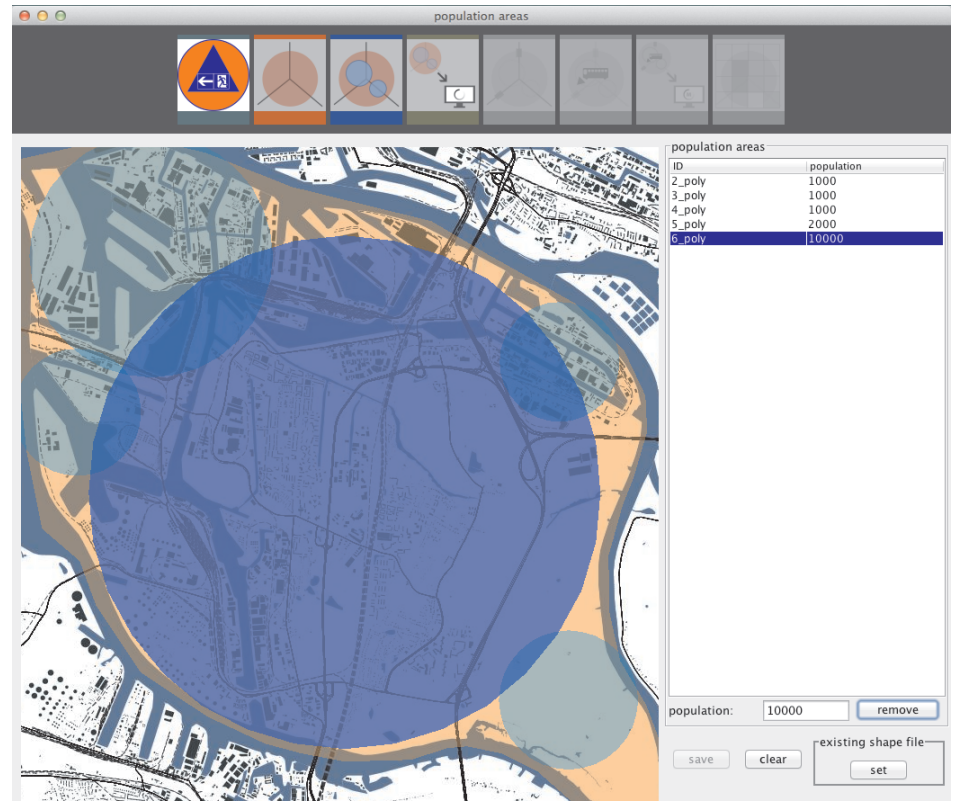

(b) Population distribution.

Figure 41.2: The evacuation area and the population distribution can be defined with an integrated GIS application.

an arbitrary number of those circles and define population figures individually for each circle. Figure 41.2 illustrates the population editor. The population editor offers only basic functionality to define a population distribution. For every circular area, the ScenarioManager produces as many agents as required and assigns each agent a random coordinate inside the circular area. However, 
in MATSim agents depart on links, so the ScenarioManager calls the getNearestLink() method defined in NetworkImpl. Thus, agents will depart on links inside and possibly near the circular areas.

In the current version, it is impossible to use a predefined demand for the simulation. Extending the simulation package in this way would be straightforward, but is out of this work's scope.

\subsubsection{Road Closures}

In real situations, some evacuation roads might not be available for the evacuation, because:

- They might be impassable due to the event (often the case in flooding-related evacuations).

- The authorities might want to keep roads open only for action/help traffic.

- In some situations, like hurricane evacuations, lane direction on motorways might be reversed to increase flow capacity in one direction.

- The authorities have detailed evacuation plans in place, with pre-planned evacuation routes; road closures might be necessary to force evacuees onto certain routes.

The actual planning of road closures can be a complex undertaking; not all attributes can be integrated into a simple tool for rapid evacuation planning. Nevertheless, the ScenarioManager offers a tool to create time-dependent road closures. An illustration of the road closures editor is given in Figure 41.3(a).

Road closures are stored as NetworkChangeEvents and handled as time-dependent network attributes in MATSim (Lämmel et al., 2010).

\subsubsection{Bus Stop Editor}

Usually, not everyone has access to a private car. In the event of an evacuation, those people often rely on public transport. In regions prone to natural disasters, local authorities normally have detailed evacuation plans in place, probably including evacuation by public transport. Consequently, it is important to have a tool available to help integrate public transport into to the simulation scenario. The ScenarioManger offers this possibility by defining bus stops and bus schedules in the interactive GUI. Figure 41.3(b) gives an example of the bus stop editor. In addition to location, the user can define when the first bus will serve a bus stop, how many buses overall will serve this particular bus stop and these buses' capacity. The Scenariomanager transforms the inputs made into the GUI into a MATSim compatible transport schedule, enriching the scenario while using the same simulation model. Details about public transport simulations with MATSim are given in Chapter 16. A tutorial can be found on the MATSim webpage http: //matsim.org/docs/tutorials/transit.

Limitations of the public transport evacuation approach in this project are:

- Each bus serves one and only one bus stop, perhaps a realistic assumption.

- Buses always take the shortest path from their designated bus stops to the safe area. As the shortest path is not necessarily the fastest, this approach might lead to avoidable delays. Some newer research investigates optimization of bus lines with respect to traffic demand and traffic conditions (Neumann, 2014). Implementing such optimization techniques in the evacuation context is a topic of future research. 


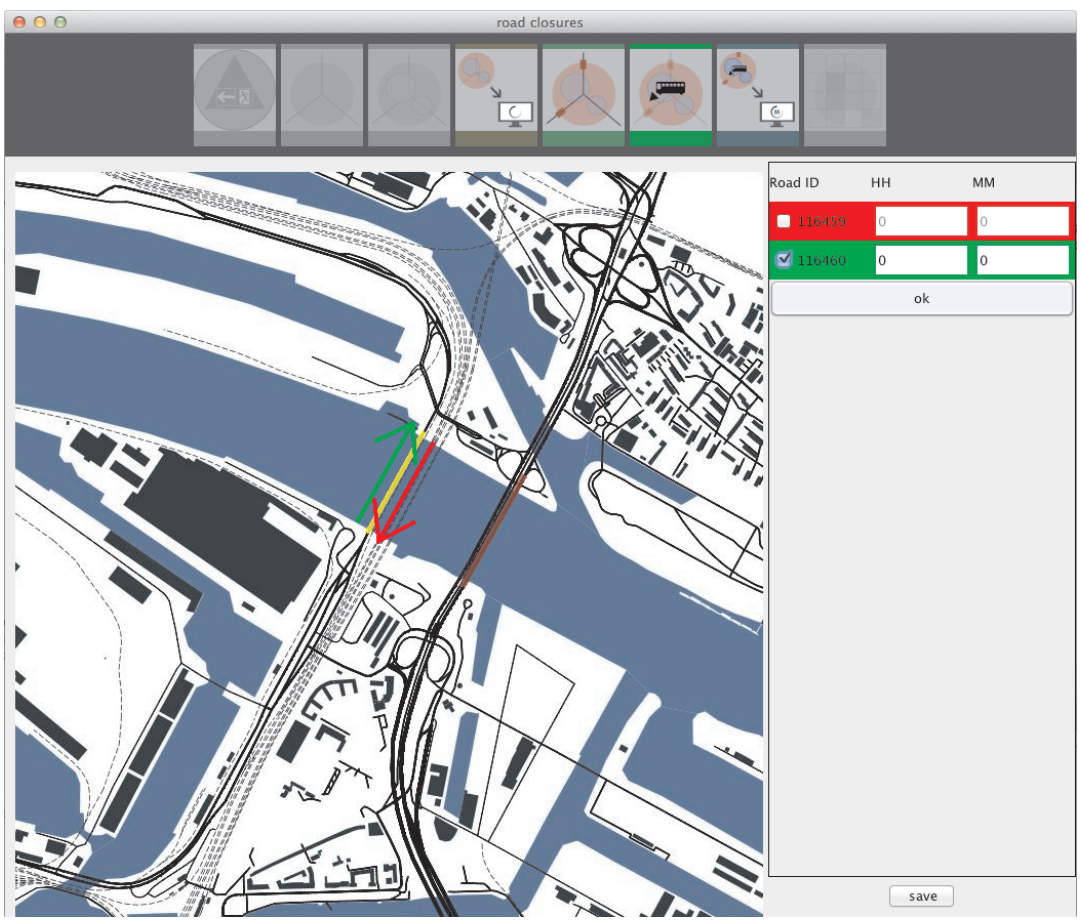

(a) Road closures.
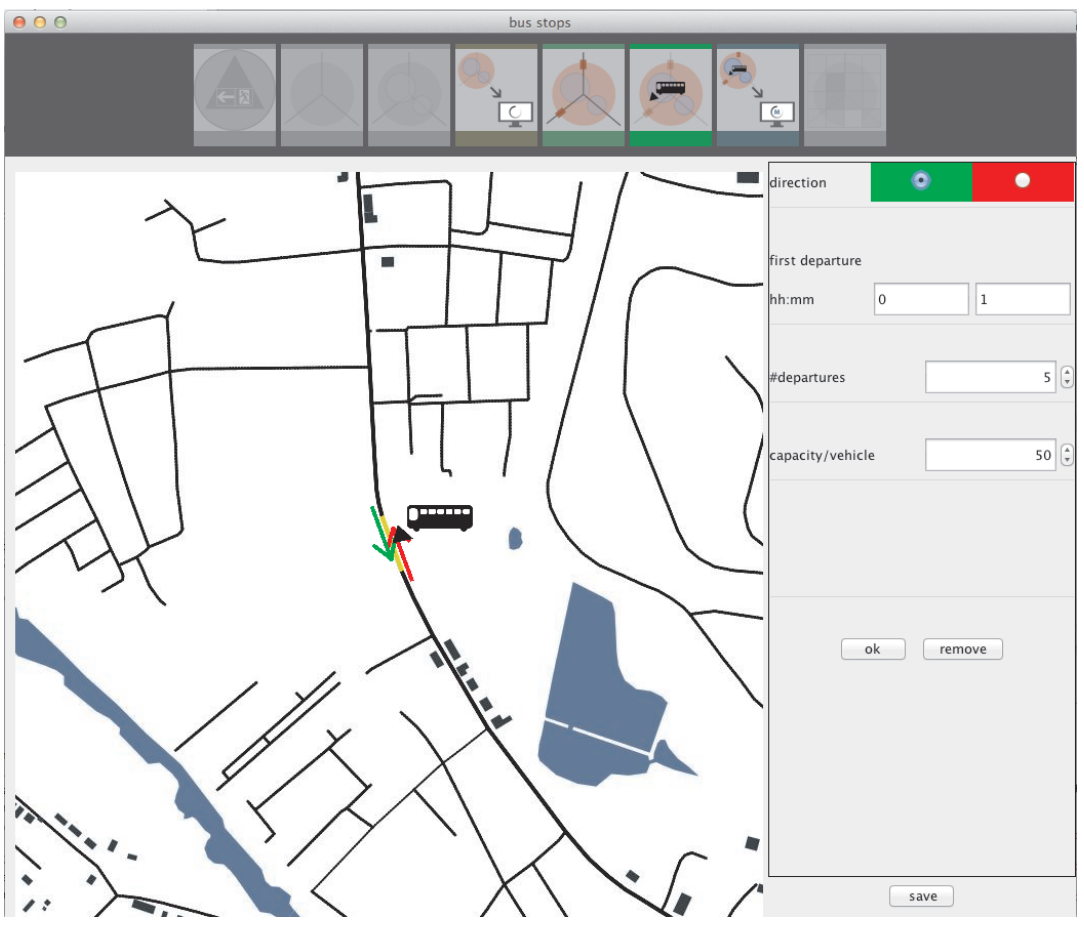

(b) Bus stop locations and schedules.

Figure 41.3: Top: Road closures can be edited by an integrated GIS application. For every link the direction and the time of closure can be defined. Bottom: Tool that let the user define bus stop locations and schedules. 


\subsubsection{Running the Scenario}

The ScenarioManager runs the evacuation simulation in a way similar to other transport simulation studies with MATSim. At the beginning, an evacuation plan is assigned to each evacuee. An evacuation plan describes how the evacuee intends to reach the safe area. If the evacuee leaves by car or on foot, the plan is essentially comprised of a route (typically the shortest) from home to the safe area. For evacuees who are depart by public transportation, the plan can be much more complex. All these evacuation plans will be executed in the mobility simulation; after this terminates, all plans are scored by travel time. The shorter a plan's travel time is, the higher is the score it receives. After this step, evacuees' plans are revised; some will receive new plans, while others continue with the current ones. This step is called re-planning. Mobility simulation, scoring, and re-planning are repeated in a loop for a predefined number of iterations; evacuees' individual performance improves over the iterations. In general transport studies, this approach emulates real-world travelers' behavior when they perform their daily commutes and try to find better travel alternatives. Evacuations, however, are singular events where such day-to-day re-planning would not occur. We argue here that the chosen iterative learning approach could be seen as the evacuees' anticipation of the conditions expected during an evacuation. People familiar with their surroundings would probably avoid roads that obviously constitute bottlenecks during an evacuation. Nevertheless, far more research is needed to definitively answer how people choose evacuation routes, or how many learning iterations are required to realistically reflect assumed anticipation skills adequately. As a rule of thumb, running 100 learning iterations are usually sufficient to achieve results constituting a lower evacuation times boundary.

\subsubsection{Analysis}

After the last iteration has finished, the ScenrioManager enables the analysis module. The analysis model evaluates the performed simulation run, using a number of different methods.

- The cumulative arrival curve tells the user the number of persons evacuated over time. From this curve, the user can, for example, learn at what time $50 \%$ of the population has reached a safe destination.

- The GIS-based evacuation time analysis draws a grid over the evacuation area and computes, for every grid cell, average evacuation time. The evacuation times are indicated by different colors; the analysis modules run a quantiles-based clustering analysis for each cell. The size of cells can be varied by the user.

- The GIS-based clearance time analysis is performed in the same way as the evacuation time analysis. The clearance time of a cell is the time when the last evacuee leaves that cell. This evacuee is not necessarily the one who also started his/her evacuation inside the corresponding cell, but might also be one who crosses that cell somewhen during the evacuation.

- A similar quantiles-based clustering approach is used for the link utilization analysis. The link utilization analysis results help the user to identify the major evacuation routes.

The analyses can be run for every single iteration for which the MATSim Controler has dumped an events file (every 10th iteration by default). An overview of the analysis module is given in Figure 41.4

\subsection{Conclusion}

This chapter demonstrates how rapid evacuation planning can be performed with help of the evacuation contribution. The evacuation contribution provides an interactive GUI to perform this task. The only required external input is a network file extracted from OSM, thus a simple scenario 


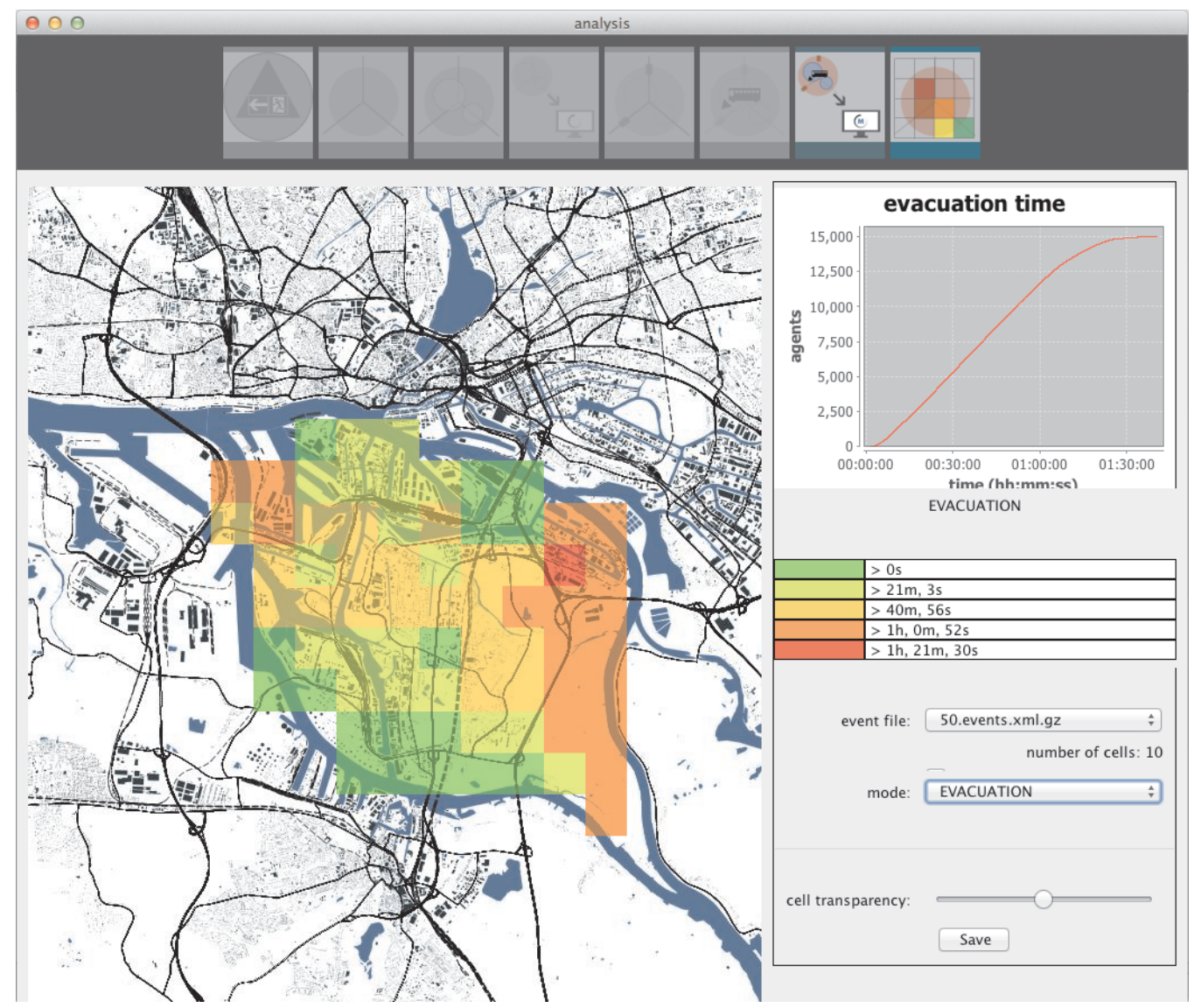

Figure 41.4: Screenshot of the analysis module showing GIS-based evacuation time analysis and the evacuation curve.

can be setup, simulated, and analyzed in less than an hour. Obviously, for an in-depth evacuation analysis of a certain area, a sort of expert knowledge is needed that a simple GUI can not supply. Still, for a rapid appraisal and for demonstration purposes, evacuation offers a powerful and easy-to-use tool. In the future, we plan to integrate a more advanced public transport planning tool based on Neumann (2014). Work is also ongoing to develop a more sophisticated pedestrian simulation model based on the theoretical framework given in Flötteröd and Lämmel (2015). 
